DR. FAYE SHARPLEY (Orcid ID : 0000-0002-5908-0645)

Article type : Original Article

\title{
Cytomegalovirus reactivation after bortezomib treatment for multiple myeloma and light chain amyloidosis
}

Faye A. Sharpley, $\dagger^{1}$ De-Silva D, $\dagger^{4}$ Shameem Mahmood, ${ }^{1,4}$ Sajitha Sachchithanantham, ${ }^{1,4}$ Ramsay Isobel, ${ }^{2}$ Garcia Mingo A, ${ }^{2}$ Sarah Worthington, ${ }^{4}$ Derralynn Hughes, ${ }^{3}$ Atul Mehta, ${ }^{3}$ Charalampian Kyriakou, ${ }^{4}$ Paul D. Griffiths, ${ }^{2}+$, Ashutosh D.Wechalekar ${ }^{1,4}+$

${ }^{1}$ National Amyloidosis Centre, University College London, London, UK

${ }^{2}$ Department of Virology, Royal Free London NHS Foundation Trust, UK

${ }^{3}$ Department of Haematology, Royal Free London NHS Foundation Trust, London, UK

${ }^{4}$ Department of Haematology, University College London Hospitals, London, UK

$\dagger$ Joint first authors

† Joint last authors

Author contributions: PG, SW and ADW conceived the study. DD, FAS and AW analysed the data and wrote the manuscript. All authors contributed to the development of the study and manuscript; and provided critical input in the manuscript.

Author correspondence:

Professor Ashutosh Wechalekar,

National Amyloidosis Centre,

University College London (Royal Free Campus)

Rowland Hill Street

This article has been accepted for publication and undergone full peer review but has not been through the copyediting, typesetting, pagination and proofreading process, which may lead to differences between this version and the Version of $\underline{\text { Record. Please cite this article as doi: 10.1111/EJH.13366 }}$

This article is protected by copyright. All rights reserved 
London, UK

Phone: 02074332758

Fax: 02074332817

a.wechalekar@ucl.ac.uk

Running title: CMV reactivation with bortezomib treatment

Word count: abstract-197, main text-2460, references-23, tables-1, figures-1

Acknowledgements: we would like to thank all the patients who participated in this study.

\begin{abstract}
:
Objective

Cytomegalovirus (CMV) is an opportunistic herpesvirus and reactivation of infection is possible in immunocompromised patients. Historically, the risk for haematology patients is restricted to those treated with an allogeneic transplant, or T-cell depleting agents. Bortezomib is a highly efficacious proteasome inhibitor widely used to treat multiple myeloma and light chain (AL) amyloidosis patients. The objective of this small prospective study was to quantify the risk of CMV reactivation associated with bortezomib treatment.
\end{abstract}

\title{
Methods
}

Fifty-seven consecutive multiple myeloma or AL amyloidosis patients commencing bortezomib-based therapy were included. Viral copy numbers were established at baseline and then at fortnightly intervals during treatment. Pre-emptive anti-viral treatment was initiated in patients with a viral load greater than 7500 copies $/ \mathrm{ml}$.

\section{Results}

Reactivation of CMV was detected in $39 \%(n=12 / 31)$ of seropositive bortezomib treated patients compared with $0 \%$ of CMV seronegative patients. Detectable DNAemia developed during the first two cycles of treatment in $83 \%(n=10 / 12)$ patients. Anti-viral treatment was initiated in $42 \%(n=5 / 12)$, but no cases of active CMV disease were seen.

\section{Conclusion}

This article is protected by copyright. All rights reserved 
This study suggests that there is a substantial risk of CMV reactivation in CMV seropositive plasma cell dyscrasia patients treated with bortezomib.

Key words: Immunology and infectious diseases, multiple myeloma. 


\section{Introduction}

Cytomegalovirus is a DNA virus of the Herpesviridae family. In the western world up to $90 \%$ of the population are positive for IgG to CMV. ${ }^{1}$ This almost ubiquitous virus is transmitted by bodily fluids which includes blood, urine, saliva, semen and breast milk and can infect epithelial, smooth muscle, blood and endothelial cells. In the immunocompetent host, primary infection is usually asymptomatic and usually occurs at a young age, but then the virus becomes dormant, establishing lifelong latency within host cells. Host $\mathrm{T}$ and natural killer (NK) cells are essential to controlling CMV infection and in the setting of impaired cellular immunity the virus can reactivate from latency. ${ }^{2}$ Immunocompromised patients are at risk of symptomatic infection which can range from a febrile episode (temperature $>38$ degrees for at least 2 days) with neutropenia and/ or thrombocytopenia, to fatal disease which can include: pneumonia, colitis, hepatitis, retinitis, mycocarditis, central nervous system disease or pancreatitis. ${ }^{3}$ Allogeneic stem cell transplant recipients (Allo-BMT) are well recognised as patients at risk of CMV disease ${ }^{4}$ and guidelines are available for the screening, monitoring and treatment of CMV in this setting. ${ }^{4}$ The risk of symptomatic CMV disease for haematology patients treated with standard chemotherapy or an autologous stem cell transplant (ASCT) is historically considered to be low.

Bortezomib is an inhibitor of the $26 \mathrm{~S}$ proteasome widely used in the treatment of multiple myeloma and AL Amyloidosis. ${ }^{5}$ Bortezomib is associated with an increased incidence of reactivation of other herpesviruses, including varicella zoster virus (VZV) and herpes simplex virus type 1 (HSV-1). ${ }^{6}$ Bortezomib appears to reduce the lymphocyte number and alter the Th1/Th2 balance, resulting in susceptibility to infections, with significantly more viral and fungal infections in patients treated with the drug. . $^{-1}$

The objective of this small prospective study was quantify the risk of CMV reactivation associated with bortezomib treatment by prospectively measuring levels of CMV DNA in patients with newly diagnosed multiple myeloma or systemic $\mathrm{AL}$ amyloidosis treated with a bortezomib based regime.

This article is protected by copyright. All rights reserved 


\section{Methods}

The National Amyloidosis Centre (NAC) provides a tertiary referral service for patients with amyloidosis and related disorders in the UK. The referring local hospitals are the treating centres, and this includes the Royal Free Hospital. Consecutive, newly diagnosed patients with multiple myeloma or $\mathrm{AL}$ amyloidosis attending $\mathrm{NAC}$ and consented for bortezomib treatment at the Royal Free Hospital from August 2014 August 2015 were included in this study. The diagnosis of multiple myeloma was made according to established International Myeloma Working Group (IMWG) criteria. ${ }^{8} \mathrm{~A}$ diagnosis of amyloidosis was confirmed by Congo red staining of a tissue biopsy with demonstration of characteristic birefringence under cross-polarized light. The amyloid subtype was confirmed by immunohistochemistry with specific antibodies, or by mass spectrometry. ${ }^{9}$ Hereditary amyloidosis was excluded by gene sequencing as appropriate. All patients had a detailed baseline assessment of organ function with biomarker assessments and imaging, including SAP scintigraphy, where appropriate. Organ involvement was defined according to the international amyloidosis consensus criteria. ${ }^{10}$ All patients had protein electrophoresis, immunofixation and serum free light chains quantified. The presence of immunoparesis was defined as one or more immunoglobulins less than the lower limit of normal. All patients were treated with a bortezomib containing regimen, either as a single agent or in combination. The choice of regimen was at the discretion of the treating clinician, based upon the patient's organ function, comorbidities and performance status.

All patients had serologic testing for CMV-specific antibodies ( $\operatorname{IgG})$ prior to treatment. CMV testing was performed using the ARCHITECT CMV IgG assay, which is a chemiluminescent microparticle immunoassay for the qualitative and semi-quantitative determination of IgG antibodies to CMV. The limit of detection with this assay is 6$60 \mathrm{AU} / \mathrm{ml}-200-250 \mathrm{AU} / \mathrm{ml}$. Patients with $>6 \mathrm{AU} / \mathrm{ml}$ were deemed to be seropositive for CMV. The CMV viral load was measured by real-time polymerase chain reaction (RTPCR) at baseline and then every two weeks from the date of their first dose of bortezomib, regardless of symptoms. Extraction of DNA from patient whole blood was performed on the QIAsymphony, using the QIAquick PCR purification kit, and quantification of CMV DNA using the artus CMV RGQ MDx Kit. Amplification of the DNA was performed on the Rotor-Gene Q. Positive CMV DNAemia was defined as the detection of CMV DNA in whole blood with a limit for detection of 200 copies/ml $(168 \mathrm{IU} / \mathrm{ml}) .^{11}$ A single CMV viral load greater than 7500 copies $/ \mathrm{ml}$ was regarded as an 
indication for anti-viral treatment. This is higher than the 3000 copies $/ \mathrm{ml}$ established as an appropriate cut-off for pre-emptive treatment in a randomised controlled trial in allograft patients, because we did not wish to over-treat these patients who, unlike the allografts, were not expected to be profoundly immunocompromised. ${ }^{12}$ In such cases patients were treated as per British Society of Haematology guidelines, ${ }^{13}$ with oral valganciclovir (900mg twice a day) for 14 days, followed by a maintenance dose of valganciclovir (450mg twice a day) until the CMV viral load was undetectable. ${ }^{3}$

Statistical analysis was performed using SPSS (IBM Corp. Released 2012. IBM SPSS Statistics for Windows, Version 21.0. Armonk, NY: IBM Corp) and Stata (StataCorp. 2017. Approval for analysis and publication was obtained from the institutional review board at the University College London. Written consent was obtained from all patients in accordance with the Declaration of Helsinki. 


\section{Results}

Baseline patient characteristics

A total of 57 patients (38 AL amyloidosis and 19 multiple myeloma) were included. The baseline patient characteristics are detailed in Table 1. Immunoparesis was present in $77.2 \%(\mathrm{n}=44 / 57)$ of patients. CMV serology results were available in $78.9 \%$ $(n=45 / 57)$ and $68.9 \%$ of patients were CMV seropositive $(n=31 / 45,68.9 \%)$. No patients had detectable viral copies prior to treatment. All patients were treated with a bortezomib containing regimen. The majority $(92 \%, n=52 / 57)$ of patients were treated with a triplet regimen and $78.9 \%$ of patients received bortezomib, cyclophosphamide and dexamethasone (VCD), $(\mathrm{n}=45 / 57)$. Four patients were treated with a doublet $(7.0 \%$, $\mathrm{n}=4 / 57$ ), bortezomib and dexamethasone (VD) and one patient with a quadruplet regimen bortezomib, melphalan, thalidomide and prednisolone (VMTP) $(1.8 \%, \mathrm{n}=1 / 57)$.

\section{CMV reactivation}

CMV reactivation was detected during bortezomib treatment in $39 \%(n=12 / 31)$ of known seropositive patients; two further patients had detectable CMV DNAemia but with an unknown baseline CMV serostatus. The patient and treatment details of these patients are detailed in Table 1 . An equal number of myeloma $(58 \%, n=7 / 12$,$) and \mathrm{AL}$ amyloidosis $(\mathrm{N}=7 / 12,58 \%)$ patients had $\mathrm{CMV}$ reactivation, however a greater proportion of myeloma versus AL amyloidosis patients developed detectable CMV during treatment ( $37 \%, \mathrm{n}=7 / 19$ versus $18 \%, \mathrm{n}=7 / 38$, respectively). Those with CMV reactivation had proteinuria in $83 \%$ of cases $(n=10 / 12)$ and immunoparesis in $100 \%$ $(n=12 / 12)$. The patients with DNAemia were seropositive in all cases $(n=12 / 12)$; the remaining two patients had missing baseline CMV serology. No CMV DNAemia was seen in the seronegative group.

The majority of patients developed detectable DNAemia within the first two cycles of bortezomib based treatment $(25 \%, n=3 / 12$, during cycle $1 ; 58 \%, n=7 / 12$ during cycle 2). In the four remaining patients, $(25 \%, n=3 / 24)$ developed detectable CMV DNA at the end of cycle 3 and one $(8 \%, n=1 / 12)$ during cycle 4 (Fig. 1). The median time from the start of treatment to the detection of viral DNAemia was 47 days. Given the small sample size, formal statistical testing of risk factors for CMV reactivation was not performed.

CMV treatment details

This article is protected by copyright. All rights reserved 
In the 12 patients who had detectable CMV, five $(42 \%, n=5 / 12)$ reached the threshold to require pre-emptive anti-viral CMV anti-viral treatment. This corresponds to $16.1 \%$ $(5 / 31)$ of seropositive patients, and $8.8 \%$ (5/57) of patients in study. All five patients received oral valganciclovir ( $900 \mathrm{mg}$ twice a day) for 14 days, followed by a maintenance dose of valganciclovir (450mg twice a day) until the CMV viral load was undetectable. No patients were deemed to have symptoms suggestive of active CMV disease. No patients were admitted to hospital due to CMV disease, but two patients with detectable viral load required admission for management of influenza A. 


\section{Discussion}

CMV reactivation or infection has the potential for serious morbidity or mortality in patients with haematological malignancies. Whilst the role of CMV infection is well characterised in the setting of allogeneic stem cell transplantation, its importance in multiple myeloma and AL amyloidosis has not been well studied. In this small prospective study, we report that nearly $40 \%$ of CMV seropositive individuals reactivate CMV during early treatment course with bortezomib. Whilst no patients developed clinical CMV disease, pre-emptive treatment was required in $42 \%$ of patients with detectable CMV titres; this corresponds to $16.1 \%$ (5/31) of seropositive patients, and $8.8 \%(5 / 57)$ of patients in study. This suggests that bortezomib is associated with a risk of CMV reactivation which warrants further evaluation.

The risk of CMV reactivation is associated with both the degree of immunosuppression and, specifically, the degree of T-cell depletion. ${ }^{14}$ Bortezomib is a highly efficacious proteasome inhibitor widely used to treat multiple myeloma and AL amyloidosis patients. A consequence of treatment is diminished cellular immunity, in particular the proliferation and function of CD8+ T lymphocytes and NK cells, ${ }^{15}$ resulting in susceptibility to infections. ${ }^{7}$ The risk of reactivation of herpesviruses, including VZV and HSV-1, is well recognised. ${ }^{6}$ In the APEX study, bortezomib treatment was associated with a significantly higher incidence of herpes zoster compared with dexamethasone treatment $(13 \%, n=42 / 331$ versus $5 \%, n=15 / 332$ respectively; $P=$ 0.0002). ${ }^{16}$ This study resulted in a change in clinical practice with the routine use of prophylactic acyclovir for the majority of patients treated with bortezomib. The exact mechanism of bortezomib induced zoster reactivation remains debatable.

CMV is also a herpesvirus, and so there is a similar theoretical risk of CMV reactivation associated with bortezomib treatment. The absence of routine monitoring does, however, make CMV reactivation difficult to diagnose and the risk of reactivation difficult to quantify. There have been a scattering of case reports of CMV reactivation in patients treated with bortezomib based regimes. ${ }^{17}$ An increased risk of CMV reactivation has also been reported in patients treated with bortezomib induction followed by an ASCT. ${ }^{18,19} \mathrm{Kim}$ et. al (2012) retrospectively evaluated 104 patients with multiple myeloma treated with ASCT with an overall CMV reactivation rate of $30.8 \%$ $(n=32 / 104)$, and $48.5 \%(n=32 / 66)$ of CMV-seropositive patients developed detectable 
CMV DNA. Patients who received conditioning therapy with melphalan, bortezomib, dexamethasone, and thalidomide were significantly more likely to develop CMV reactivation $(\mathrm{P}=0.015) .{ }^{19}$ A prospective study performed by Marchesi et. al (2013) of 80 patients with multiple myeloma treated with ASCT also reported a significantly higher rate of CMV reactivation in patients who received bortezomib and immunomodulatory therapy when compared to standard anthracycline based treatment ( $9.4 \%$ versus $1.1 \% \mathrm{p}=0.019)$, but not in patients treated with immunomodulatory therapy alone. The study concluded that patients treated with bortezomib-based regimens were at higher risk of developing symptomatic CMV reactivation after ASCT.

${ }^{20}$ A more recent retrospective study performed by Hasegawa et.al (2016) of 120 patients with multiple myeloma reported a CMV infection rate of $20 \%$ and three cases of CMV disease. This study included patients who did not proceed to ASCT, and $80 \%$ of the patients with CMV reactivation were treated with bortezomib. ${ }^{21}$

A comprehensive literature review performed by Marchesi et al (2017) of nontransplant haematology patients revealed highly-variable CMV reactivation rates ranging between $2-39 \%,{ }^{20}$ but this included a wide range of agents including bortezomib, bendamustine and rituximab. The CMV reactivation rate of $39 \%$ of seropositive patients in this current study is similar to the $48.5 \%$ reported by Kim et.al 19; although these studies are not directly comparable due to different patient populations. The current data clearly shows that CMV reactivation in the non-transplant setting is under-recognised.

Little is known about the risk of CMV reactivation with time/ duration of treatment. In this current study the majority of CMV reactivation events occurred during the first two cycles of bortezomib treatment. Hasegawa et al (2016) reported a median duration from diagnosis to CMV reactivation of 5 months (1-86 months) in CMV seropositive patients, with a significantly longer interval in CMV negative patients, where the median was 20 months (1-84 months) $(\mathrm{P}=0.025)$. This suggests that $\mathrm{CMV}$ reactivation may be an event which occurs during the course of treatment, although further studies are require to confirm this finding.

Factors predicting CMV reactivation also remain unclear. In our study, which included patients with systemic AL amyloidosis, there appeared to be higher proportion of patients with immunoparesis $(n=44,77 \%)$ and proteinuria $(n=41,72 \%)$. This was also reflected in the patients who developed detectable CMV (immunoparesis, $n=12 / 12$, 
$100 \%$; proteinuria, $n=10 / 12,83 \%$ ). Given the small number of patients, formal statistical testing of risk factors for CMV reactivation was not possible.

Other studies have reported the presence of extramedullary disease and low absolute neutrophil count as risk factors, ${ }^{21}$ but this may well simply be a marker of extensive pre-treatment.

The current data suggests that reactivation is the major cause of CMV DNAemia since none of the seronegative patients developed detectable CMV DNA. No patients developed CMV disease, but, due to use of a pre-emptive treatment strategy, this study is unable to infer how many patients would have developed CMV end-organ disease without treatment. However, in the study by Hasegawa et al (2016), where there was no pre-emptive treatment strategy, $66 \%$ of patients developed clinically significant CMV disease. The lack of CMV disease in this study suggests one of two interpretations: that the CMV reactivation rate is significant in patients treated with bortezomib, but that the risk of CMV disease is low, as suggested by previous guidelines ${ }^{22}$ and/or that preemptive anti-viral treatment is an effective strategy to prevent symptomatic infection.

This study is not without limitations. This was a small study with AL and myeloma patients and so formal statistical testing was not possible. The baseline CMV serology was known in all but two cases; we cannot therefore fully exclude primary infection in these two cases. Patients were also pre-emptively treated, hence the true incidence of clinical CMV disease remains unclear. Despite these limitations, this study confirms that there is a risk of CMV reactivation in patients treated with bortezomib. The clinical significance of these reactivations requires further study, particularly as more complex triplet or quadruplet regimes, with greater consequent immunosuppression, are being used to treat patients with multiple myeloma and AL amyloidosis. The significance of CMV reactivation for patients who are treated with bortezomib, and then subsequently receive an autologous stem cell transplant, also requires further exploration. Future studies should also include hepatitis B serology given the possibility of hepatitis B virus as a risk factor for CMV reactivation. ${ }^{23}$ Given the difficulty of clinically recognising $\mathrm{CMV}$ infection, the current findings also raises an important issue of CMV viral monitoring, which must be addressed in further prospective studies in larger patient 
cohorts. Physicians must also remain alert to the possibility of CMV infection with bortezomib treatment in patients with relevant atypical infective symptoms.

Acknowledgements: we would like to thank all the staff at the National Amyloidosis centre and the patients who participated in this study.

Conflict of Interest: the authors have no competing interests to declare. 
Table 1: Summary patient characteristics, including those with cytomegalovirus reactivation

\begin{tabular}{|c|c|c|}
\hline & $\begin{array}{l}\text { All patients }(\% / \\
\text { range }), \mathrm{n}=\mathbf{5 7}\end{array}$ & $\begin{array}{l}\text { CMV reactivated } \\
\text { patient } n=14\end{array}$ \\
\hline \multicolumn{3}{|l|}{ Diagnosis } \\
\hline AL amyloidosis & 38 & 7 \\
\hline Multiple Myeloma & 19 & 7 \\
\hline \multicolumn{3}{|l|}{ Gender } \\
\hline Male & 33 & 9 \\
\hline Female & 24 & 5 \\
\hline Age, years, (median/range) & $63(33-89)$ & $63(37-78)$ \\
\hline \multicolumn{3}{|l|}{ Paraprotein Isotype } \\
\hline None & 31 & 6 \\
\hline $\operatorname{IgG}$ & 16 & 4 \\
\hline $\operatorname{IgA}$ & 5 & 3 \\
\hline $\operatorname{IgM}$ & 3 & 1 \\
\hline $\operatorname{IgD}$ & 1 & 0 \\
\hline $\operatorname{IgG}$ and $\operatorname{Ig} \mathrm{A}$ & 1 & 0 \\
\hline Paraprotein g/L (median/ range) & $20.5(1.1-97)$ & $38.5(3-97)$ \\
\hline FLC ratio (range) & $<0.01-6166.7$ & $<0.01-6166.7$ \\
\hline FLC mg/L (median/range) & $1924.5(28.1-16000)$ & $867(103-9250)$ \\
\hline \multicolumn{3}{|l|}{ Immunoparesis } \\
\hline Yes & 44 & 12 \\
\hline No & 13 & 2 \\
\hline \multicolumn{3}{|l|}{ Proteinuria } \\
\hline Yes & 41 & 10 \\
\hline No & 13 & 3 \\
\hline Unevaluable & 6 & 1 \\
\hline \multicolumn{3}{|l|}{ CMV serostatus pre-treament } \\
\hline Positive & 31 & 12 \\
\hline Negative & 14 & 0 \\
\hline Unevaluable & 12 & 2 \\
\hline \multicolumn{3}{|l|}{ Chemotherapy regimen } \\
\hline Bortezomib +steroids (VD) & 3 & 0 \\
\hline Bortezomib+steroids: & & \\
\hline - + Alkylator (VCD/VMP/ VC/ VCP) & 48 & 12 \\
\hline - + IMiD (VTD/ VRD/ VMTP/ VCTD) & 5 & 1 \\
\hline
\end{tabular}

This article is protected by copyright. All rights reserved 


\begin{tabular}{|l|l|l|}
\hline$\bullet+$ Anthacycline (PAD) & 1 & 1 \\
\hline
\end{tabular}

CMV, cytomegalovirus; AL, light chain amyloidosis; Ig, immunoglobulin; FLC, free light chain; VCD, velcade, cyclophosphamide, dexamethasone; VD, velcade, dexamethasone; VRD, velcade, lenalidomide, dexamethasone; VTD, velcade, thalidomide, dexamethasone; VC, velcade, cyclophosphamide; VCTD, velcade, cyclophosphamide, thalidomide, dexamethasone; VCP, velcade, cyclophosphamide, prednisolone; VMTP, velcade, melphalan, thalidomide, prednisolone; PAD, Bortezomib, doxorubicin, dexamethasone. 


\section{Figure legends:}

Figure 1: Course of DNAemia in 14 patients with detectable CMV following bortezomib-based therapy 


\section{References}

1. Zuhair M, Smit GSA, Wallis G, et al. Estimation of the worldwide seroprevalence of cytomegalovirus: A systematic review and meta-analysis. Reviews in medical virology. 2019;29(3):e2034.

2. Sylwester AW, Mitchell BL, Edgar JB, et al. Broadly targeted human cytomegalovirus-specific CD4+ and CD8+ T cells dominate the memory compartments of exposed subjects. The Journal of experimental medicine. 2005;202(5):673-685.

3. Ljungman P, Griffiths P, Paya C. Definitions of cytomegalovirus infection and disease in transplant recipients. Clinical infectious diseases : an official publication of the Infectious Diseases Society of America. 2002;34(8):10941097.

4. Ljungman $\mathrm{P}$, Hakki M, Boeckh M. Cytomegalovirus in hematopoietic stem cell transplant recipients. Hematology/oncology clinics of North America. 2011;25(1):151-169.

5. Adams J. Development of the proteasome inhibitor PS-341. The oncologist. 2002;7(1):9-16.

6. Oakervee HE, Popat R, Curry N, et al. PAD combination therapy (PS341/bortezomib, doxorubicin and dexamethasone) for previously untreated patients with multiple myeloma. British journal of haematology. 2005;129(6):755-762.

7. Li J, Li Y, Huang B, Zheng D, Chen M, Zhou Z. Drug-induced modulation of T lymphocytes as a potential mechanism of susceptibility to infections in patients with multiple myeloma during bortezomib therapy. Cell biochemistry and biophysics. 2015;71(1):457-464.

8. Rajkumar SV, Dimopoulos MA, Palumbo A, et al. International Myeloma Working Group updated criteria for the diagnosis of multiple myeloma. The Lancet Oncology. 2014;15(12):e538-548.

9. Rezk T, Gilbertson JA, Mangione PP, et al. The complementary role of histology and proteomics for diagnosis and typing of systemic amyloidosis. J Pathol Clin Res. 2019.

This article is protected by copyright. All rights reserved 
10. Gertz MA, Comenzo R, Falk RH, et al. Definition of organ involvement and treatment response in immunoglobulin light chain amyloidosis $(\mathrm{AL})$ : a consensus opinion from the 10th International Symposium on Amyloid and Amyloidosis, Tours, France, 18-22 April 2004. American journal of hematology. 2005;79(4):319-328.

11. Ljungman $\mathrm{P}$, Boeckh $\mathrm{M}$, Hirsch $\mathrm{HH}$, et al. Definitions of Cytomegalovirus Infection and Disease in Transplant Patients for Use in Clinical Trials. Clinical infectious diseases : an official publication of the Infectious Diseases Society of America. 2017;64(1):87-91.

12. Griffiths PD, Rothwell E, Raza M, et al. Randomized Controlled Trials to Define Viral Load Thresholds for Cytomegalovirus Pre-Emptive Therapy. PloS one. 2016;11(9):e0163722.

13. Emery V, Zuckerman M, Jackson G, et al. Management of cytomegalovirus infection in haemopoietic stem cell transplantation. British journal of haematology. 2013;162(1):25-39.

14. van Burik JA, Carter SL, Freifeld AG, et al. Higher risk of cytomegalovirus and aspergillus infections in recipients of T cell-depleted unrelated bone marrow: analysis of infectious complications in patients treated with $\mathrm{T}$ cell depletion versus immunosuppressive therapy to prevent graft-versus-host disease. Biology of blood and marrow transplantation : journal of the American Society for Blood and Marrow Transplantation. 2007;13(12):1487-1498.

15. Pellom ST, Jr., Dudimah DF, Thounaojam MC, Sayers TJ, Shanker A. Modulatory effects of bortezomib on host immune cell functions. Immunotherapy. 2015;7(9):1011-1022.

16. Chanan-Khan A, Sonneveld P, Schuster MW, et al. Analysis of herpes zoster events among bortezomib-treated patients in the phase III APEX study. Journal of clinical oncology : official journal of the American Society of Clinical Oncology. 2008;26(29):4784-4790.

17. Hioki T, Takama H. Cytomegalovirus reactivation accompanied by varicella zoster virus reactivation or reinfection in an adult patient of multiple myeloma during bortezomib therapy. 2018;45(1):108-109.

18. Marchesi F, Mengarelli A, Giannotti F, et al. High incidence of post-transplant cytomegalovirus reactivations in myeloma patients undergoing autologous

This article is protected by copyright. All rights reserved 
stem cell transplantation after treatment with bortezomib-based regimens: a survey from the Rome transplant network. Transplant infectious disease : an official journal of the Transplantation Society. 2014;16(1):158-164.

19. Kim JH, Goulston C, Sanders S, et al. Cytomegalovirus reactivation following autologous peripheral blood stem cell transplantation for multiple myeloma in the era of novel chemotherapeutics and tandem transplantation. Biology of blood and marrow transplantation : journal of the American Society for Blood and Marrow Transplantation. 2012;18(11):1753-1758.

20. Marchesi F, Pimpinelli F, Ensoli F, Mengarelli A. Cytomegalovirus infection in hematologic malignancy settings other than the allogeneic transplant. Hematological oncology. 2018;36(2):381-391.

21. Hasegawa T, Aisa Y, Shimazaki K, Ito C, Nakazato T. Cytomegalovirus reactivation in patients with multiple myeloma. European journal of haematology. 2016;96(1):78-82.

22. Ljungman $\mathrm{P}$, de la Camara R, Cordonnier C, et al. Management of CMV, HHV-6, HHV-7 and Kaposi-sarcoma herpesvirus (HHV-8) infections in patients with hematological malignancies and after SCT. Bone marrow transplantation. 2008;42(4):227-240.

23. Zekri AR, Mohamed WS, Samra MA, Sherif GM, El-Shehaby AM, El-Sayed MH. Risk factors for cytomegalovirus, hepatitis $B$ and $C$ virus reactivation after bone marrow transplantation. Transplant immunology. 2004;13(4):305-311.

This article is protected by copyright. All rights reserved 


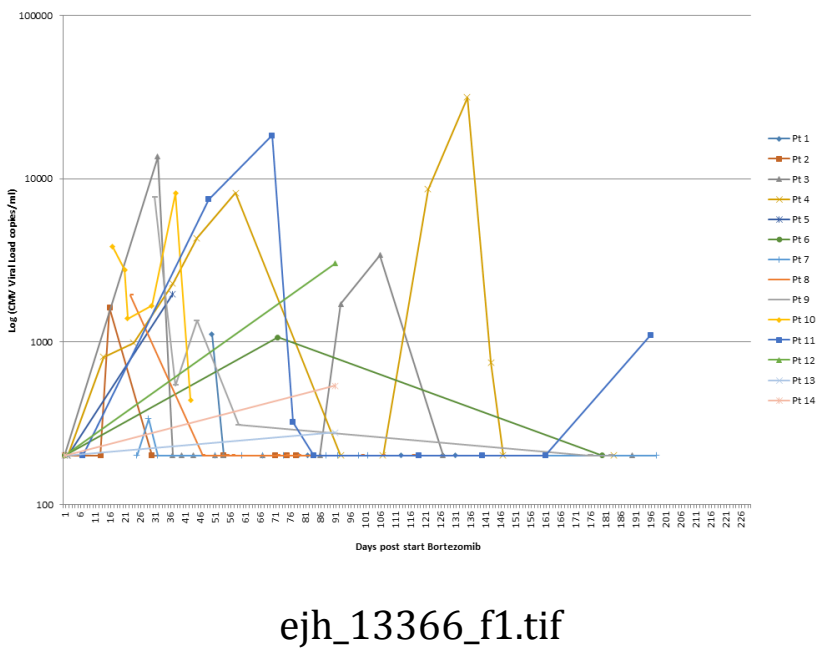

This article is protected by copyright. All rights reserved 